\title{
REALIDADE VIRTUAL E TECNOLOGIA ASSISTIVA: AMBIENTE SEGURO PARA TREINAMENTO DE CADEIRANTES CONTROLADO POR SINAIS ELETROMIOGRÁFICOS
}

\author{
D. P. Salgado*, F.R. Martins*, L. Borges* e E.L.M. Naves* \\ *Núcleo de Tecnologias Assistivas - Faculdade de Engenharia Elétrica \\ Universidade Federal de Uberlândia, Uberlândia, Brasil. \\ e-mail: deborapsalgado@gmail.com
}

\begin{abstract}
Resumo: O trabalho proposto baseia-se no projeto de controle de uma cadeira de rodas virtual por meio de sinais de eletromiografia de superfície (sEMG) desenvolvido por Silva (2013) [1], sendo uma versão modular em uma plataforma mais flexível e atualizada. Além disso, este módulo faz parte de um sistema integrado para controle multimodal de uma cadeira de rodas. O objetivo é de que o módulo seja uma ferramenta de treinamento para usuários que venham a utilizar o acionamento por sEMG numa cadeira de rodas motorizada (CRM) real de forma autônoma e segura. A metodologia usada baseia-se na utilização de Realidade Virtual (RV) para desenvolver uma Tecnologia Assistiva (TA) e, para tanto, foi utilizado um ambiente virtual desenvolvido na plataforma Unity para desenvolver a interface e o controle do sistema. A aquisição do sinal de entrada foi feita através de um sensor EMG acoplado a um microcontrolador, com o processamento e filtragem do sinal feito de forma embarcada.
\end{abstract}

Palavras-chave: tecnologia assistiva, realidade virtual, sinais de eletromiografia, sistema embarcado.

Abstract: This work aims to control a virtual wheelchair through surface electromyography signals (sEMG) developed by Silva (2013) [1], being a modular version on a platform that is more flexible and up to date. In addition, this module is part of an integrated system for multimodal control of a wheelchair. The objective is to make the module a training tool for users who will use the sEMG to control a real electricpowered wheelchair (EPW) autonomously and safely. The method used is based on Virtual Reality (VR) to develop an Assistive Technology (AT) and, therefore, it was used a virtual environment created in Unity to develop the interface and system control. The acquisition of the input signal was made using an EMG sensor coupled to a microcontroller with embedded signal processing and filtering.

Keywords: assistive technology, virtual reality, electromyographic signals, embedded system.

\section{Introdução}

A Organização Mundial da Saúde (OMS) divulgou um estudo em 2011 que revela que $15 \%$ da população mundial apresentam algum tipo de deficiência e que 70 milhões de pessoas precisam de cadeiras de rodas [2]. Segundo o censo do IBGE, o Brasil possui 45 milhões de deficientes e em torno de 9 milhões de pessoas possuem algum tipo de dificuldade motora. Dentro deste número, 3,7 milhões sofrem com dificuldade motora severa [3]. É esperado para os próximos anos que a incidência de deficientes aumente em função do envelhecimento das populações e o risco de deficiência na população com idade avançada.

$\mathrm{O}$ relatório da OMS evidencia que essas pessoas apresentam as piores perspectivas de saúde, níveis mais baixos de escolaridade, participação econômica menor e taxas de pobreza mais elevada se comparado com pessoas hígidas. Essa realidade se deve ao fato das barreiras enfrentadas por eles, que incluem discriminação, ausência de serviços de reabilitação, mobilidade e infraestrutura sem acessibilidade. Para atingir perspectivas melhores e mais duradouras, novas tecnologias mais acessíveis (barata e de alta qualidade) são desenvolvidas pela motivação de tornar a vida do usuário cada vez mais fácil e que seja disponível a todas as classes sociais.

Sendo assim, diante desta realidade, é crescente a demanda por dispositivos de tecnologia assistiva (TA). Dessa forma, ao auxiliar esses indivíduos, os dispositivos de TA promovem acessibilidade, autonomia, reinserção na sociedade, aumento da autoestima e melhoria de qualidade de vida dos mesmos. TA é uma área que integra áreas interdisciplinares para desenvolver dispositivos, recursos, serviços, práticas, entre outros facilitadores que proporcione uma ampliação de uma habilidade funcional deficitária que possibilite a realização de uma função que se encontra impedida por circunstância de deficiência.

Dispositivos de TA que possibilitam a mobilidade ao usuário são um dos pilares para reinserção desses indivíduos na sociedade. Existem hoje para auxílio à mobilidade bengalas, muletas, andadores, carrinhos, cadeiras de rodas manuais ou elétricas, scooters e cadeiras de rodas inteligentes (smartwheelchairs). Infelizmente, existe um alto índice de pessoas que não consegue utilizar ferramentas manuais, ou que em algum determinando momento terão que abandonar tais 
ferramentas em função do agravamento da deficiência, por exemplo, em casos em que o indivíduo perderá a maioria de seus movimentos voluntários, como tetraplegia e esclerose lateral amiotrófica. Como solução, os sinais bioelétricos são recursos possíveis para controlar um dispositivo de TA. Um exemplo é o sinal eletromiográfico (EMG) onde o usuário pode expressar sua intenção através de contrações musculares [5].

A cadeira de rodas motorizada (CRM) é um dispositivo de TA indispensável para auxílio à mobilidade de pessoas com deficiências motoras severas. Entretanto, alguns usuários apresentam dificuldades em conduzi-las de forma adequada e testes com cadeiras de rodas reais podem envolver riscos à segurança [6]. A partir dessa problemática, nota-se a necessidade do uso de treinamento para utilização da TA de forma segura. A solução seria utilizar a tecnologia da realidade virtual (RV) para desenvolvimento de uma ferramenta para treinamento do uso da TA. Na área saúde, a RV é bastante aplicada para reproduzir situações reais sem risco a pacientes, pela possibilidade de simular e visualizar ações impossíveis de serem percebidas no mundo real [7], como ferramenta para treinamento, reabilitação e educação, entre outros [8].

Considerando os avanços da tecnologia e a existência de uma plataforma flexível de desenvolvimento, este projeto se insere no cenário de tecnologias assistivas com a proposta de desenvolver uma interface de comando de uma cadeira virtual baseada em sinal eletromiográfico. A ferramenta tem o propósito de ser utilizada como treinamento para usuários que venham a utilizar uma cadeira motorizada real com sistema de controle por EMG.

\section{Materiais e métodos}

Para o projeto de sistema de controle da cadeira virtual por sEMG (sinais eletromiográficos de superfície), primeiramente foi construído um diagrama representando o conceito abstrato das interligações entre as plataformas utilizadas no sistema. Na Figura 1 é possível se ver quais são os dados de entrada, o fluxo lógico de funcionamento e qual é a resposta na saída.

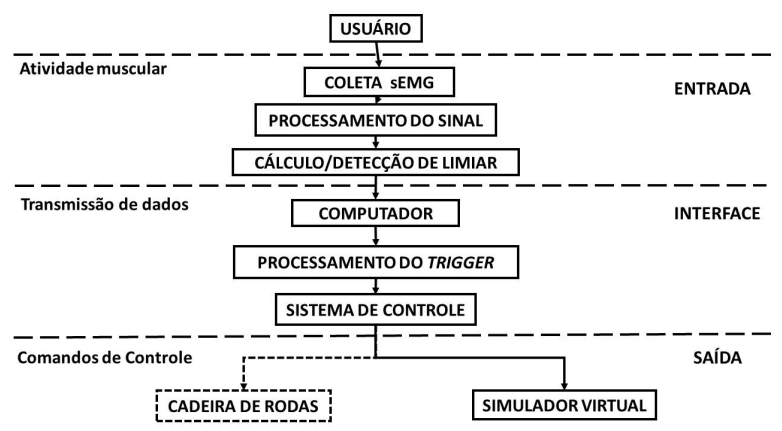

Figura 1: Diagrama de Blocos Detalhado do Sistema de Controle da Cadeira Virtual por sEMG.
A implementação do sistema proposto envolveu as etapas a seguir:

Definição dos materiais para coleta do sinal de eletromiografia - Para a coleta do sinal de eletromiografia foram utilizados eletrodos de superfície passivos de $\mathrm{Ag} / \mathrm{AgCl}$ em configuração bipolar. A utilização dessa configuração é uma tentativa de eliminar interferências oriundas de outras atividades biológicas do paciente ou da rede elétrica, já que por meio da utilização de um amplificador diferencial com alta taxa de rejeição de modo comum (CMRR), qualquer ruído comum aos dois eletrodos deve ser atenuado.

O sensor escolhido foi o Myoware $\AA$ da fabricante Adafruit. O sensor em questão detecta a atividade elétrica muscular, em seguida converte-a para uma voltagem variável que é lida no pino de entrada analógico de um microcontrolador. Além de atender aos requisitos mínimos para aplicação do projeto, o Myoware tem duas opções de saída, o sinal bruto e o sinal encapsulado (envoltória do sinal). Essa característica do sensor oferecer à envoltória do sinal evita a necessidade de algumas de processamento do sinal.

Definição do funcionamento do módulo de controle por sinal sEMG - São colocados no usuário os sensores especificados anteriormente. A detecção do sinal de eletromiografia de superfície é feita em um músculo localizado na face. No projeto foi utilizado apenas um canal EMG como entrada. Foram préselecionados os músculos masseter e occipitofrontal, de forma que os eletrodos posicionados em algum destes músculos captassem a atividade de contração dos mesmos. O sinal captado é filtrado, processado e transformado em um comando do tipo trigger (gatilho) para uma interface apresentada para o usuário. Para o processamento do sinal, foi utilizado um processo de detecção limiar dinâmico, calculado a partir de uma janela de 200 amostras coletadas e armazenadas no microcontrolador.

Processamento do sinal EMG - O sensor é ligado ao Arduino (constituído por um microcontrolador do modelo ATmega328 que possui um conversor A/D de 10 bits com precisão de \pm 2 LSBs), onde o sinal é digitalizado, processado e transmitido para a interface de controle do simulador virtual. Após a conversão do sinal analógico para o digital é iniciada a rotina de processamento do sinal. No final do processamento é identificada a ação do usuário (neste caso se houve contração muscular ou não).

Se a ação do usuário foi uma contração o processador envia um trigger (do tamanho de dois bytes do tipo char) para o sistema de controle do simulador. $\mathrm{O}$ processamento do sinal foi feito de forma embarcada, no qual o computador é completamente dedicado ao dispositivo (ao sistema que ele controla). Deste modo, é possível aperfeiçoar o projeto reduzindo custos, tamanho e recursos computacionais do projeto.

Definição da interface de controle para a cadeira virtual - O módulo de controle da cadeira virtual foi 
desenvolvido com o objetivo de ser utilizado como forma de treinamento, avaliação e calibração do sistema da cadeira real. Sendo assim, é obrigatório que exista uma correspondência do ambiente virtual com o real, ou seja, a interface do simulador deve ser o mais próximo do que se encontra no sistema de controle da cadeira de rodas real, e os mesmos comandos de controle são utilizados para ambos os ambientes, real e virtual. Em paralelo ao projeto proposto, está sendo desenvolvido um sistema de controle por módulo sEMG para uma cadeira de rodas real. Deste modo, a interface de controle para cadeira de rodas virtual foi desenvolvida de forma semelhante à interface para cadeira de rodas real.

O controle da cadeira funciona a partir de um princípio de seleção indireta por varredura linear, ou seja, as opções vão sendo exibidas uma a uma até que o usuário faça sua escolha. Um primeiro gatilho detectado no músculo é usado para seleção do movimento desejado (frente, ré, esquerda, direita e pare). Assim que seja selecionado o item desejado pelo usuário, este recebe então um feedback visual indicado qual comando foi selecionado. Este comando é então enviado para a cadeira, que irá executá-lo até um comando diferente seja recebido. Toda vez que um comando é selecionado o próximo comando esperado da cadeira é o de "pare". Na Figura 2 e Figura 3 é possível ver a seleção do comando "frente" logo em seguida o próximo comando esperado é de "pare".

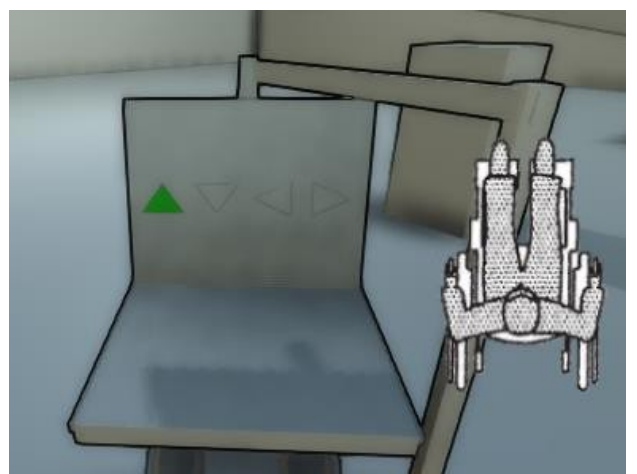

Figura 2: Interface de controle desenvolvida para a cadeira de rodas virtual, primeira tela.

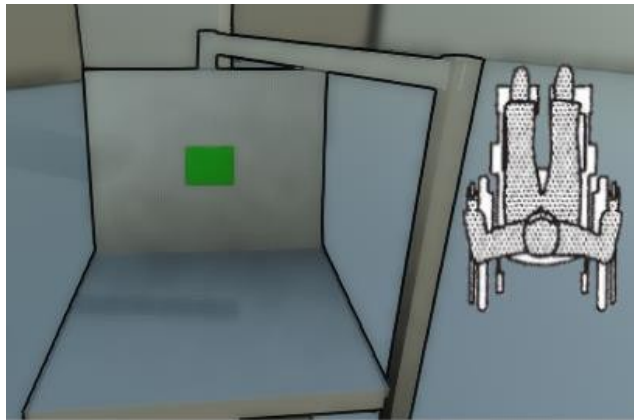

Figura 3: Interface de controle desenvolvida para a cadeira de rodas virtual, segunda tela.

\section{Resultados}

Após o desenvolvimento do módulo de controle da cadeira virtual por sEMG da face, obteve-se um programa de processamento embarcado baseado na linguagem $\mathrm{C} / \mathrm{C}++$, uma interface gráfica e um algoritmo em C\# para o controle da cadeira do simulador, chamado uViEW. Foram feitos testes nos músculos do masséter e do occipitofrontal. Ambos os músculos são localizados na face uma vez que o objetivo do presente trabalho é fornecer controle do simulador para pessoas com grave deficiência motoras. O dispositivo de entrada é composto pelo sensor Myoware, microntrolador e o firmware para processamento do sinal. A comunicação entre o dispositivo de entrada e a interface é verificada pelo algoritmo de controle da cadeira (Figura 4), e o teste final do projeto é mostrado na Figura 5.

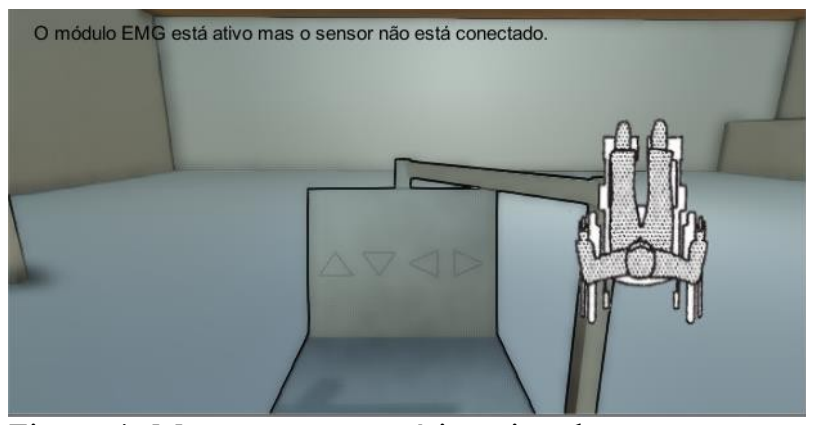

Figura 4: Mensagem ao usuário avisando que o sensor está desconectado.

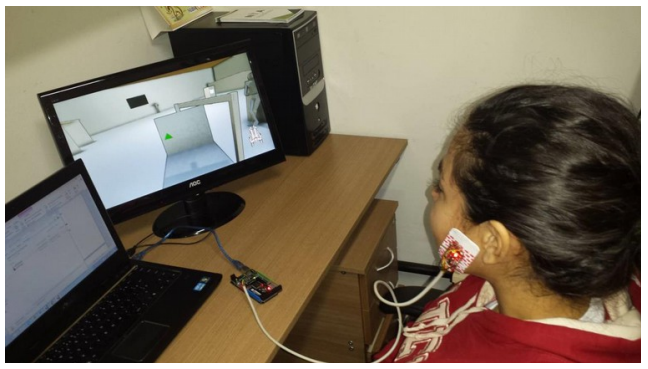

Figura 5: Teste do projeto.

\section{Discussão}

Nos primeiros testes realizados, o voluntário conseguiu conduzir a cadeira de rodas virtual conforme proposto. Os comandos foram passados de forma rápida para ambiente virtual que proporcionou uma resposta e u m feedback satisfatório para o usuário. Entretanto, durante a fase de testes com outros voluntários percebeu-se a necessidade de desenvolver um sistema de calibração do limiar de contração.

Uma vez que o sistema de detecção funciona perfeitamente com algumas pessoas e com outras não desempenha de forma plena, foi identificado problemas de detecção de contração duplicada e de falta de reconhecimento de contração em determinados momentos do teste, ou seja, casos em que o limiar estaria abaixo e acima do ideal, respectivamente. Dessa forma, o sistema calibração ajudaria a detectar características especificas do sinal cada usuário, 
ajustando a detecção de contração de forma mais precisa. Verificou-se que a comunicação serial, entre os dispositivos de entrada e o simulador, teve um bom desempenho, não apresentando problemas durante os testes.

O sistema de controle foi desenvolvido para que o usuário pudesse controlar a cadeira de forma simplificada, com menor número de contrações possíveis e assim menor gasto energético. Desta forma, o módulo utiliza apenas um canal EMG diferente do trabalho anterior [1] que utilizou dois canais, e o sistema de seleção dos comandos da cadeira foi desenvolvido baseado no princípio de seleção indireta por varredura linear. Para trabalhos futuros, o método precisa passar por um processo de validação para saber se o sistema pode ser utilizado por mais tempo sem gerar fadiga muscular, sendo necessários testes mais longos (com duração maior que 20 minutos) que não realizados neste trabalho.

\section{Conclusão}

O presente trabalho criou a oportunidade para o aprendizado de áreas específicas dentro da Engenharia Biomédica, e por ser um trabalho com conhecimentos da área de Tecnologia Assistiva e Realidade Virtual, o trabalho proporcionou contato com tecnologias emergentes da área e um melhor entendimento da mesma.

Neste trabalho, foi desenvolvido um módulo de controle para uma cadeira virtual por sinais de eletromiografia de músculos faciais. $\mathrm{O}$ módulo apresenta uma interface de controle que funciona com o princípio de seleção indireta por varredura linear. A interface mostrou-se capaz de operar a cadeira virtual com o uso do sensor, porém apresentou dificuldade para detectar contração muscular em alguns testes. Por usar um método de seleção indireta, o sistema funciona a partir de um gatilho, e dessa forma não são necessários dois músculos para interagir com ele fazendo com que o treinamento seja feito de forma simplificada.

\section{Agradecimentos}

Os autores agradecem ao CNPq, a CAPES e a FAPEMIG pelo apoio financeiro necessário para a realização da pesquisa proposta.

\section{Referências}

[1] A. N. Silva, Y. Morere, E. L. M. Naves, A. A. R. De $\mathrm{Sa}$, and A. B. Soares, "Virtual electric wheelchair controlled by electromyographic signals," ISSNIP Biosignals Biorobotics Conf. BRC, pp. 1-5, 2013.

[2] OMS, Relatório mundial sobre a deficiência. 2011.

[3] CENSO, "Cartilha do Censo 2010: Pessoas com Deficiência." 2010.

[4] R. Bersch, "Introdução À Tecnologia Assistiva," p. 29, 2013.
[5] C. G. J. Pinheiro, E. L. M. Naves, P. Pino, E. Losson, A. O. Andrade, G. Bourhis, P. J. C.G., N. E.L., P. P., L. E., and A. A.O., "Alternative communication systems for people with severe motor disabilities: a survey," Biomed. Eng. Online, vol. 10, p. 31, 2011.

[6] L . Fehr, W. E. Langbein, and S. B. Skaar, "Adequacy of power wheelchair control interfaces for persons with severe disabilities: a clinical survey.," J. Rehabil. Res. Dev., vol. 37, no. 3, pp. 353-60, 2000.

[7] F. de L. dos S. Nunes, R. M. E. M. da Costa, L. dos S. Machado, and R. M. de Moraes, "Realidade virtual para saúde no brasil: Conceitos, desafios e oportunidades," Rev. Bras. Eng. Biomed., vol. 27, no. 4, pp. 243-258, 2011.

[8]J. Sanchez, S. Cobb, P. Sharkey, and J. Merrick, "Virtual reality and assistive technologies for people with disabilities," Int. J. Disabil. Hum. Dev., vol. 10, no. 4, pp. 275-276, 2011. 